\title{
Aparición simultánea de cáncer gástrico temprano y GIST
}

\section{Simultaneous appearance of early gastric cancer and GIST}

Martín Alonso Gómez Zuleta, ${ }^{\star}$ Javier Humberto Riveros Vega, ${ }^{2}$ Óscar Fernando Ruiz Morales. ${ }^{3}$

\footnotetext{
1 Especialista en Gastroenterología, Universidad Nacional de Colombia, Especialista en Medicina Interna, Universidad Nacional de Colombia, Hospital Universitario Nacional, Hospital Occidente de Kennedy, Unidad de gastroenterología y Ecoendoscopia (UGEC). Bogotá D. C., Colombia.

2 Especialista en Gastroenterología, Universidad Nacional de Colombia, Especialista en Medicina Interna, Pontificia Universidad Javeriana, Hospital Occidente de Kennedy, Hospital Universitario Nacional de Colombia. Bogotá D. C., Colombia. ${ }^{3}$ Especialista en Gastroenterología, Universidad Nacional de Colombia, Especialista en Medicina Interna, Universidad San Martín, Hospital Occidente de Kennedy, Hospital Universitario Nacional de Colombia. Bogotá D. C., Colombia.
}

*Correspondencia: magomez@unal.edu.co.

Fecha recibido: $\quad 30 / 01 / 18$

Fecha aceptado: 20/06/18

\begin{abstract}
Resumen
Se presenta el caso clínico de un paciente masculino de 74 años quien ingresó con síntomas de sangrado digestivo alto, se realizó una endoscopia de vías digestivas altas y se encontraron dos lesiones en el estómago: una elevada de centro ulcerado y otra subepitelial, lo que llevó a completar los estudios con una ecoendoscopia gástrica que mostró una lesión mucosa que infiltraba la submucosa sugestiva de cáncer gástrico temprano y otra lesión subepitelial dependiente de la muscular, sugestivas de un tumor gastrointestinal estromal (GIST); los estudios de estatificación no evidenciaron compromiso metastásico, por lo que se llevó al manejo quirúrgico con la posterior confirmación histológica de los hallazgos.
\end{abstract}

\section{Palabras clave}

Hematemesis, melenas, cáncer gástrico, tumor gastrointestinal estromal, GIST, tumor sincrónico, ki 67, cirugía, ecoendoscopia, disección submucosa, gastrectomía.

\begin{abstract}
We present the case of a 74-year-old male patient who was admitted with symptoms of upper digestive bleeding. Endoscopy of his upper digestive tract found an ulcerated lesion and a subepithelial lesion in his stomach. Complete studies including gastric endoscopic ultrasound showed a mucosal lesion infiltrating the submucosa which was suggestive of early gastric cancer as well as a subepithelial lesion on the muscle that was suggestive of a gastrointestinal stromal tumor (GIST). Staging showed no metastatic compromise, so surgery was performed, and histology subsequently confirmed the findings.
\end{abstract}

\section{Keywords}

Hematemesis, manes, gastric cancer, gastrointestinal stromal tumor, GIST, synchronous tumor, ki 67, surgery, endoscopic ultrasound, submucosal dissection, gastrectomy.

\section{INTRODUCCIÓN}

El cáncer gástrico (CG) es un tumor que tiene una alta prevalencia y alta tasa de mortalidad a nivel mundial. En el 2008 se produjeron 988000 casos nuevos de CG en el mundo, por lo que representa el cuarto cáncer más frecuente después del cáncer de pulmón, cáncer de seno y cáncer de colon y recto; y se produjeron 738000 muertes, lo que constituye la segunda causa de muerte por cáncer (1). En Colombia, es la primera causa de muerte por cáncer en hombres y la tercera causa de muerte por cáncer en las mujeres (1). La endoscopia digestiva es el método de diagnóstico de elección para el CG (2). La ultrasonografía endoscópica (USE) es el método complementario de elección para determinar la profundidad del CG temprano (3). Tiene la capacidad de visualizar los estratos del tubo 
digestivo con una correlación histológica demostrada (3). Los CG tempranos están localizados en la mucosa y submucosa, con o sin compromiso de nódulos linfáticos (4), este tipo de CG se trata endoscópicamente, mediante mucosectomía o disección endoscópica de la submucosa, dependiendo del tamaño y las características morfológicas, determinadas mediante la USE (5). El CG avanzado es aquel que en profundidad invade más allá de la submucosa y que tiene compromiso metastásico regional y a distancia (5), el manejo de este incluye la cirugía y el tratamiento oncológico con quimio y radioterapia.

Los tumores gastrointestinales estromales (GIST) son los tumores gastrointestinales más comunes del tracto gastrointestinal (6), con una incidencia entre 10 y 15 casos por millón, usualmente su diagnóstico es incidental en estudios radiológicos o endoscópicos y su manifestación clínica más frecuente es el sangrado gastrointestinal (5). La localización más frecuente es el estómago (7); desde el punto de vista histológico, más del $95 \%$ de GIST son positivos para la proteína KIT (CD117) y cerca del $90 \%$ presenta mutación en el gen c-KITo en el gen PDGFRA (8). En la endoscopia se puede ver como una lesión subepitelial en ocasiones con una ulceración central (9) y en la USE se encuentra como una lesión hipoecogénica, homogénea, dependiente de la capa muscular, utilizándose este estudio de forma complementaria para realizar la toma de biopsias guiadas con el fin de llegar al diagnóstico histológico (10). La tomografía axial computarizada (TAC) es la imagen de elección para caracterizar una masa a nivel abdominal ya que evalúa su extensión local y a distancia, debido a que el GIST puede presentar compromiso metastásico en especial en el hígado, epiplón y cavidad peritoneal (11).

El manejo depende de la extensión y del tamaño, el tratamiento quirúrgico busca una resección con márgenes libres, la linfadenectomía no es necesaria en vista de que el compromiso linfático es raro (11), el $40 \%$ a $50 \%$ de los pacientes llevados a cirugía pueden presentar recurrencia (12), por lo que los inhibidores de la tirosina-cinasa aparecen como una excelente alternativa para el tratamiento (13). La resección en cuña es el manejo quirúrgico de elección (14), la técnica de laparoscopia al ser comparada con la resección abierta ha demostrado menores complicaciones, tiempo de estancia hospitalaria y sangrado (15); el tratamiento de las lesiones menores de $2 \mathrm{~cm}$ no es claro al momento de valorar la evidencia disponible, por lo que a menos que se documente extensión a distancia, lo cual es raro, el manejo es expectante (16).

En este artículo se presenta un caso de las dos patologías expuestas previamente, con un punto interesante, ya que ambas aparecen de forma sincrónica en el paciente.

\section{CASO CLÍNICO}

Paciente de 74 años quien ingresó por cuadro de 3 días de evolución de hematemesis y melenas, se realizó una endoscopia de vías digestivas altas y se encontró a nivel del cuerpo medio hacia la pared anterior una lesión elevada de $20 \mathrm{~mm}$ de diámetro con centro ulcerado, también se encontró otra lesión de aspecto subepitelial de $60 \mathrm{~mm}$ a nivel del antro; el diagnóstico endoscópico inicial fue elevación gástrica en estudio tipo 0-IIa/0-IIc y lesión subepitelial en un estudio (iGIST?) (Figura 1). Se tomaron múltiples biopsias de las lesiones.

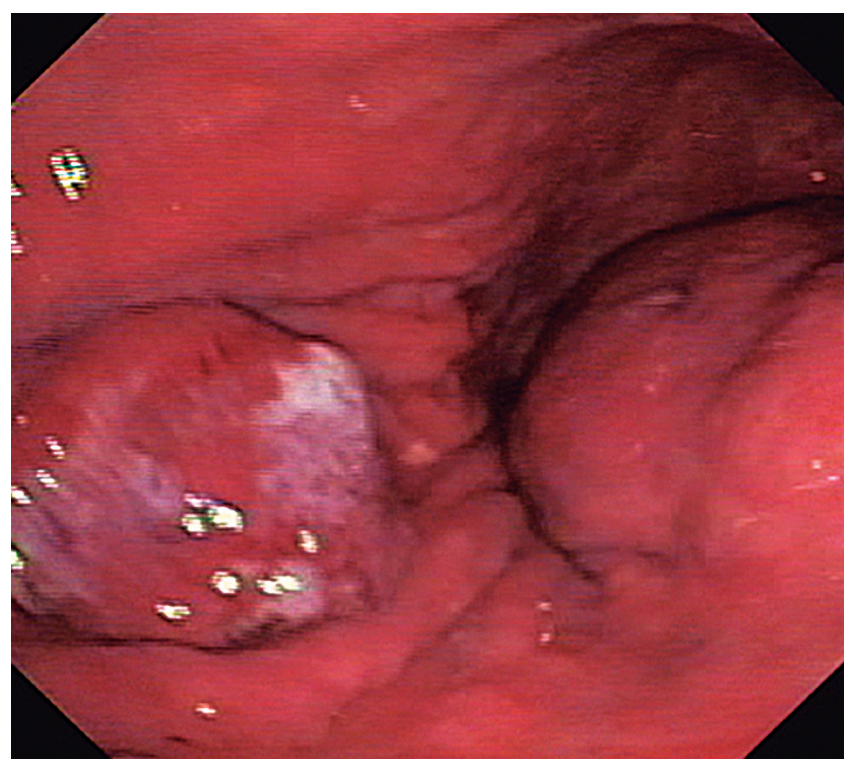

Figura 1. En la imagen se ven las dos lesiones, la de la izquierda corresponde al cáncer gástrico temprano y la de la derecha corresponde al GIST.

Teniendo en cuenta los hallazgos endoscópicos, se decidió ampliar el estudio a través de una ultrasonografía endoscópica gástrica, la cual mostró en el cuerpo una lesión elevada de $20 \mathrm{~mm}$ hipoecoica que infiltraba la mucosa y parcialmente la submucosa, en el antro hubo otra lesión subepitelial, la cual dependía de la muscular propia de $60 \mathrm{~mm}$ de diámetro con espacios quísticos en su interior (Figura 2). No se encontraron adenopatías perilesionales o en el tronco celíaco, se realizó el diagnóstico de CG temprano y GIST en la cuarta capa. La biopsia tomada a la lesión del cuerpo gástrico confirmó la presencia de un adenocarcinoma gástrico moderadamente diferenciado. Se realizó un TAC abdominal contrastado y se evidenció únicamente que en el GIST no había metástasis.

Se planeó realizar una disección submucosa del adenocarcinoma y resección quirúrgica del GIST, pero el paciente presentó un sangrado agudo por ulceración del GIST y fue llevado a gastrectomía subtotal con resección de las dos 
lesiones. La patología del espécimen quirúrgico mostró un adenocarcinoma moderadamente diferenciado con extensión únicamente a la submucosa superficialmente (Figura 3) y lesión antral de $7 \times 7 \mathrm{~cm}$ a la cual se le realizó inmunohistoquímica positiva para c-kit (Figura 4), CD 34 positiva y $\mathrm{S} 100$ negativo, con un índice mitótico bajo $<2 \%$ y un ki $67<2 \%$ (Figura 5). Todos los ganglios resultaron negativos para lesión. La evolución del paciente es muy satisfactoria actualmente ( 1 año de seguimiento) y está asintomático; dado que el CG era temprano y que el GIST era de bajo riesgo no necesitó un tratamiento complementario.

\section{DISCUSIÓN}

La ocurrencia de GIST con otras neoplasias no es frecuente, hay algunos reportes de casos así como series de casos que han mostrado esta relación. Krame y colaboradores (17) mostraron en una serie de casos una mayor frecuencia

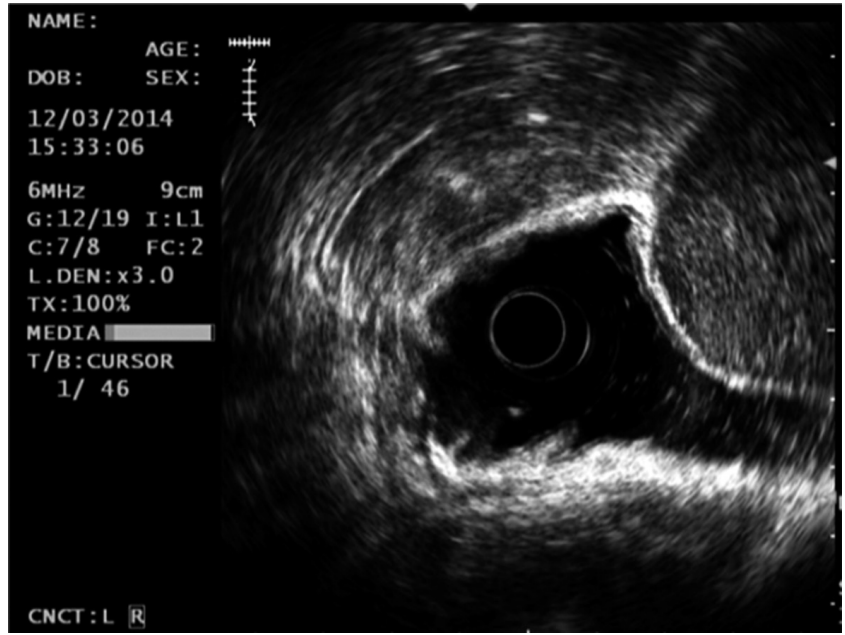

Figura 2. Ultrasonografía endoscópica gástrica. A la izquierda, CG; a la derecha, lesión subepitelial.

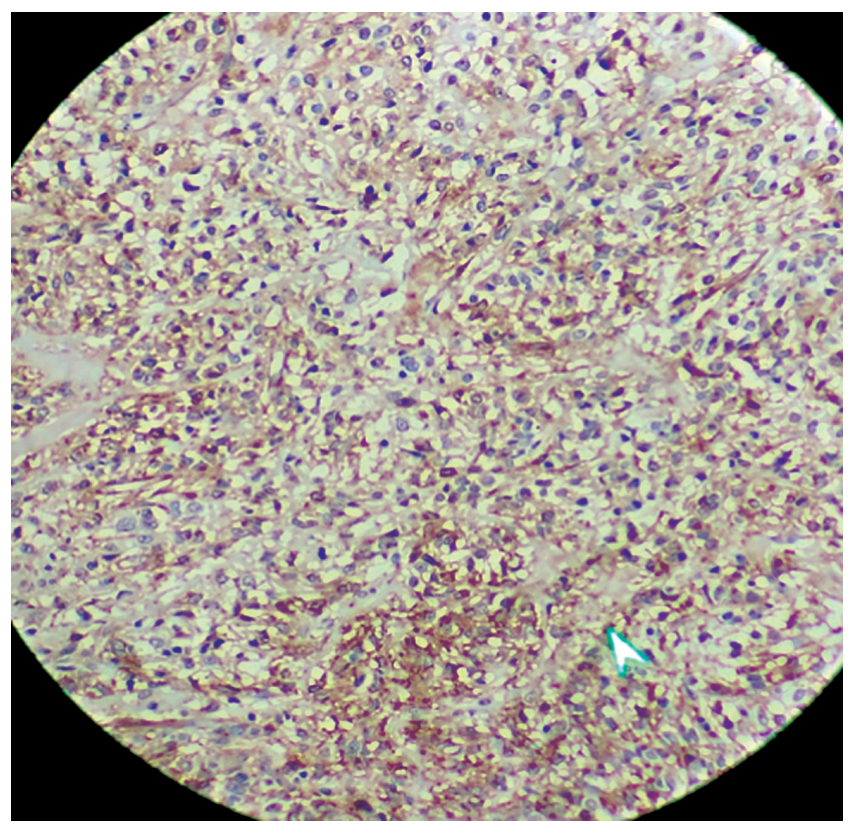

Figura 4. GIST c-kit positivo.

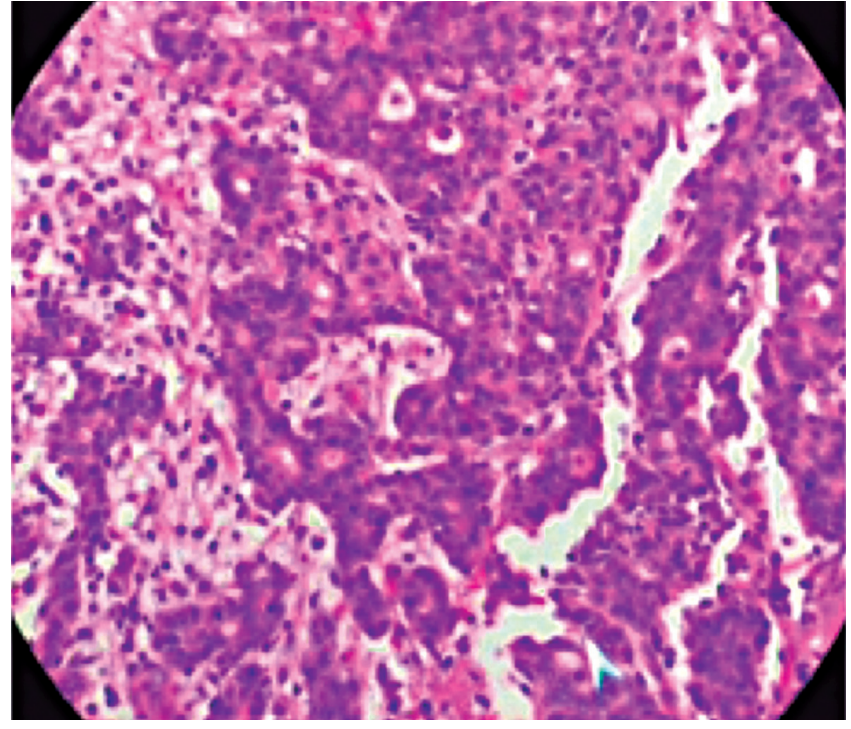

Figura 3. Adenocarcinoma gástrico moderadamente diferenciado.

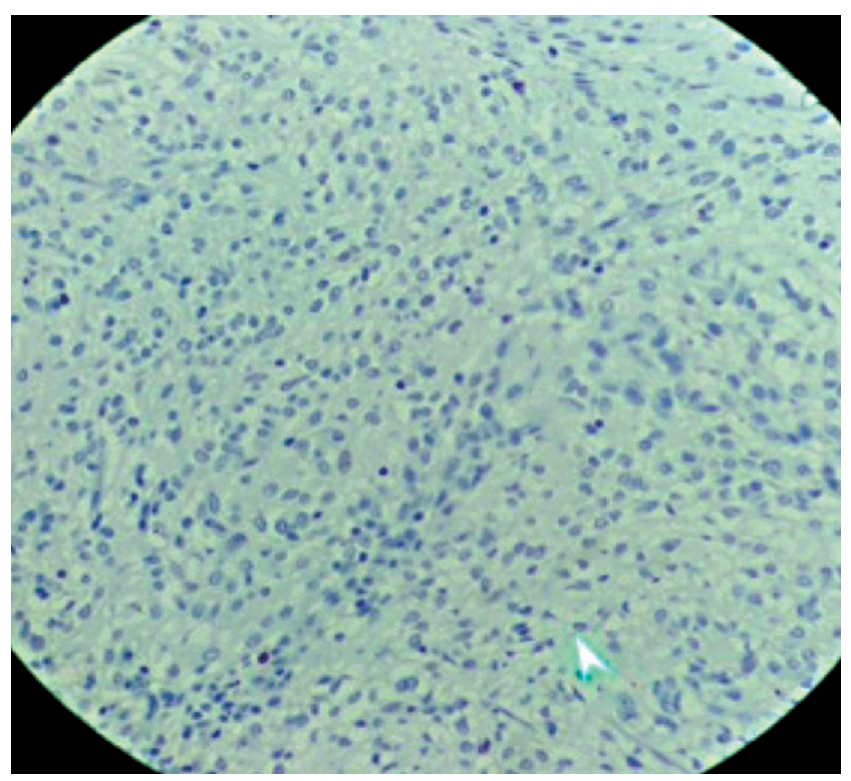

Figura 5. GIST CD $34(+)$ S100 (-). 
de diferentes tipos de tumores en pacientes que tenían o habían padecido GIST, en 836 pacientes con GIST encontraron $31,9 \%$ de otros tipos de neoplasias, gastrointestinales en un $43,5 \%$, urogenitales y de mama en el $34,1 \%$, hematológicas en 7,3\% y de piel en el 7,3\%; sin embargo, la mayoría de estas se encontró hasta 5 años después del diagnóstico de GIST sin describir neoplasias sincrónicas. En otra serie por Goncalves (18) de 101 pacientes se encontró un $13,8 \%$ con otros tipos de tumores; de estos, un $57,1 \%$ (8 casos) con CG, ninguno de ellos sincrónico.

De esta forma, siendo ya conocida la relación del GIST con la presencia de otras neoplasias, la presentación sincrónica es muy infrecuente, apenas encontrada en pocas series de casos, solo Wronski y colaboradores (19) mostraron 28 casos de GIST con tumores sincrónicos y encontraron que un $57 \%$ de estos eran CG. Aunque es importante aclarar que, teniendo en cuenta el tipo de estudios que describen la relación GIST con CG, no se puede describir una asociación causal ni mucho menos tomar a una u otra patología como factor de riesgo para la otra.

Así mismo, pese que hay hipótesis sobre la ocurrencia de neoplasias sincrónicas con GIST, ninguna de ellas ha sido comprobada aún. Sería útil establecer seguimientos mayores, así como estudios con mayor tamaño de muestra en los que la comparación con controles permitiera establecer una asociación de riesgo entre estas patologías, pero no deja de ser de vital importancia reportes como el que hemos presentado para ilustrar al lector sobre esta posible asociación y de este modo refinar la búsqueda de CG temprano en pacientes con GIST o a la inversa.

Adicionalmente, en vista de la evidencia disponible y su contundencia es necesario considerar como manejo de primera elección en el CG el manejo endoscópico, y el más efectivo es la disección endoscópica de la submucosa (5). Por su parte, la resección en cuña por laparoscopia es la elección más apropiada para el manejo quirúrgico de los GIST gástricos debido a su alta efectividad y menor tasa de eventos adversos (16). En el caso de este paciente, la decisión tomada de realizar gastrectomía subtotal fue guiada más por la emergencia de la hemorragia severa presentada.

\section{REFERENCIAS}

1. Ferlay J, Shin H, Bray F, Formar D, Mathers C, Parkin D. Estimates of worldwide burden of cancer in 2008: GLOBOCAN 2008. Int J Cancer. 2010;127(12):2893-917. https://doi.org/10.1002/ijc.25516.

2. Bowrey DJ, Griffin SM, Wayman J, Karat D, Hayes N, Raimes SA. Use of alarm symptoms to select dyspeptics for endoscopy causes patients with curable esophagogastric cancer to be overlooked. Surg Endosc. 2006;20(11):17258. https://doi.org/10.1007/s00464-005-0679-3.
3. Kwee R, Kwee T. Imaging in local staging of gastric cancer: a systematic review. J Clin Oncol. 2007;25(15):2107-16. https://doi.org/10.1200/JCO.2006.09.5224.

4. Tatsuta M, Iishi H, Okuda S, Oshima A, Taniguchi H. Prospective evaluation of diagnostic accuracy of gastrofiberscopic biopsy in diagnosis of gastric cancer. Cancer. 1989;63(7):1415-20.

5. Japanese Gastric Cancer Association. Japanese Classification of Gastric Carcinoma - 2nd English Edition. Gastric cancer. 1998;1(1):10-24. https://doi.org/10.1007/PL00011681.

6. Sanchez-Hidalgo JM, Duran-Martinez M, Molero-Payan R, Rufian-Peña S, Arjona-Sanchez A, Casado-Adam A, et al. Gastrointestinal stromal tumors: A multidisciplinary challenge. World J Gastroenterol. 2018;24(18):1925-41. https://doi.org/10.3748/wjg.v24.i18.1925.

7. Harlan LC, Eisenstein J, Russell MC, Stevens JL, Cardona K. Gastrointestinal stromal tumors: treatment patterns of a populationbased sample. J Surg Oncol. 2015;111(6):702-7. https://doi.org/10.1002/jso.23879.

8. Fletcher CD, Berman JJ, Corless C, Gorstein F, Lasota $\mathrm{J}$, Longley BJ, et al. Diagnosis of gastrointestinal stromal tumors: A consensus approach. Hum Pathol 2002; 33: 45965. https://doi.org/10.1053/hupa.2002.123545.

9. Tio TL, Tytgat GN, den Hartog Jager FC. Endoscopic ultrasonography for the evaluation of smooth muscle tumors in the upper gastrointestinal tract: an experience with 42 cases. Gastrointest Endosc. 1990;36(4):342-50.

10. Ando N, Goto H, Niwa Y, Hirooka Y, Ohmiya N, Nagasaka $\mathrm{T}$, et al. The diagnosis of GI stromal tumors with EUSguided fine needle aspiration with immunohistochemical analysis. Gastrointest Endosc. 2002;55(1):37-43. https://doi. org/10.1067/mge.2002.120323.

11. Everett M, Gutman H. Surgical management of gastrointestinal stromal tumors: analysis of outcome with respect to surgical margins and technique. J Surg Oncol 2008; 98: 588-593. https://doi.org/10.1002/jso.21030.

12. Dematteo RP, Gold JS, Saran L, Gönen M, Liau KH, Maki RG, et al. Tumor mitotic rate, size, and location independently predict recurrence after resection of primary gastrointestinal stromal tumor (GIST). Cancer 2008;112(3):60815. https://doi.org/10.1002/cncr.23199.

13. Gold JS, Dematteo RP. Combined surgical and molecular therapy: the gastrointestinal stromal tumor model. Ann Surg. 2006;244(2):176-84. https://doi.org/10.1097/01. sla.0000218080.94145.cf.

14. Novitsky YW, Kercher KW, Sing RF, Heniford BT. Longterm outcomes of laparoscopic resection of gastric gastrointestinal estromal tumors. Ann Surg 2006;243(6):738-45. https://doi.org/10.1097/01.sla.0000219739.11758.27.

15. Bischof DA, Kim Y, Dodson R, Carolina Jimenez M, Behman $\mathrm{R}$, Cocieru A, et al. Open versus minimally invasive resection of gastric GIST: a multiinstitutional analysis of short- and long-term outcomes. Ann Surg Oncol. 2014;21(9):2941-8. https://doi.org/10.1245/s10434-014-3733-3.

16. Balde AI, Chen T, Hu Y, Redondo N JD, Liu H, Gong W, et al. Safety analysis of laparoscopic endoscopic coopera- 
tive surgery versus endoscopic submucosal dissection for selected gastric gastrointestinal stromal tumors: a propensity scorematched study. Surg Endosc. 2017;31(2):843-51. https://doi.org/10.1007/s00464-016-5042-3.

17. Kramer K, Wolf S, Mayer B, Schmidt SA, Agaimy A, HenneBruns D, et al. Frequence, spectrum and prognostic impact of additional malignancies in patients with gastrointestinal stromal tumors. Neoplasia. 2015 Jan;17(1):134-40. https:// doi.org/10.1016/j.neo.2014.12.001.
18. Gonçalves R, Linhares E, Albagli R, Valadao M, Vilhena B, Romano $\mathrm{S}$, et al. Occurrence of other tumors in patients with GIST. Surg Oncol. 2010;19(4):140-3. https://doi. org/10.1016/j.suronc.2010.06.004.

19. Wronski M, Ziarkiewicz-Wroblewska B, Gornicka B, Cebulski W, Slodkowski M, Wasiutynski A, et al. Synchronous occurrence of gastrointes- tinal stromal tumors and other primary gastrointestinal neoplasms. World J Gastroenterol. 2006;12(33):5360. https://doi. org/10.3748/wjg.v12.i33.5360 